Dengqiu Ma

Yongping Liu $\bowtie$

Zhenhuan Ye

Yongqiao Wei

Jing Liu

https://doi.org/10.21278/TOF.451018220

ISSN 1333-1124

eISSN 1849-1391

\title{
ANALYSIS OF THE TOOTH SURFACE CONTACT AREA OF A CIRCULAR-ARC-TOOTH-TRACE CYLINDRICAL GEAR UNDER LOAD
}

\begin{abstract}
Summary
To reconstruct the tooth surface of a circular-arc-tooth-trace cylindrical gear (CATT cylindrical gear), a 3D model has been developed and the contact characteristics have been investigated. Based on the development principle and meshing theory, the tooth surface equation, tooth surface curvature equation and tooth surface contact ellipse equation of the CATT cylindrical gear were deduced, and it was proved that the contact was a point contact. Then, the tooth surface was reconstructed and a 3D model was developed. Next, by performing the finite element analysis and meshing impression experiment, it was proved again that the contact is the point contact, and the contact area became an ellipse under loading. Finally, the influences of the design parameters on the contact ellipse were investigated. The general tendency is that the elliptical contact area increases from the tooth root to the gear top; the elliptical contact area decreases when the modulus and the gear tooth number near the tooth root increase and it increases when the modulus and the gear tooth number near the tooth top increase; the elliptical contact area increases when the tooth line radius increases. The elliptical contact area decreases in a cliff-like manner near the tooth top. The research results provide a reference for the design, profile modification and lubrication of the CATT cylindrical gear.
\end{abstract}

Key words: $\quad$ circular-arc-tooth-trace cylindrical gear; tooth surface equation; contact ellipse; finite element method; tooth line radius; module and gear tooth number.

\section{Introduction}

Gears have been studied by numerous researchers for a long time [1-3]. But, the circular-arc-tooth-trace (CATT) cylindrical gear is a new kind of gear transmission. In comparison to involute spur and helical gears, CATT cylindrical gears have an arc tooth line, whose advantages are the higher contact ratio, high load carrying capacity, low sensitivity to installation errors and no axial force acting on gear teeth. These factors result in the capacity of CATT cylindrical gears to carry higher load and be more stable [4-6]. 
Since the curved tooth cylindrical gear was developed, numerous studies on the CATT cylindrical gear have been done by many researchers. F. L. Litvin [7-8] derived an ideal meshing equation of the CATT cylindrical gear and the profile equation of the conjugate tooth and contact line equation by using the gear meshing kinematic method, which laid the theoretical basis for research. Further, Tamotsu Koga [9] and Dai Yutang [10] analyzed transmission characteristics and the methods for processing CATT cylindrical gears. R. T. Tseng [11-13] developed a mathematical model of CATT cylindrical gears by applying the vector method and studied the contact characteristics and processing methods. Di Yutao and Chen Ming [5] analyzed the forming principle, meshing performance and bearing capacity of CATT cylindrical gears. Song Aiping [4, 14-15] also derived a tooth surface equation of arc gears and studied the bending stress; however, the gear was cut by a translational processing device with parallel linkage. Xiao Huajun [16] and Wang Shaojiang [17] studied the tooth surface form and performed three dimensional modelling of CATT cylindrical gears from the viewpoint of manufacture. Many other researchers also investigated contact strength, bending strength, gear transmission errors, computerized design and simulation of meshing [18-21], including the comparison of the maximum contact stress in spur gears, helical gears and CATT cylindrical gears, applying the finite element analysis as the research method.

According to current literature, the research into CATT cylindrical gears is chaotic and a complete theoretical system has not been established so far. Theoretically, the forms of contact of CATT cylindrical gears are divided into two kinds, the line contact and the point contact. But no researcher has defined the specific contact form of CATT cylindrical gears, although the contact form of CATT cylindrical gears has a major impact on gear's stability, reliability, and noise. So, the contact form must be discussed in order to facilitate the processing, design and application of CATT cylindrical gears.

In the present paper, based on the meshing theory and processing and using the space meshing theory and differential geometry, the tooth surface equation, tooth surface curvature equation and tooth surface contact ellipse equation of the CATT cylindrical gear were deduced to find the contact form of the CATT cylindrical gear. Then, the tooth surface of the CATT cylindrical gear was reconstructed and a 3D model was developed. Next, to prove the contact form additionally, the finite element analysis and the meshing impression experiment were used. Finally, the influences of the tooth line radius, module and gear tooth number on the contact ellipse were investigated. The research results provide a reference for the design, profile modification and lubrication of CATT cylindrical gears.

\section{Tooth surface equation}

The reference coordinate system of the CATT cylindrical gear was set up as shown in Fig. 1. $o_{1}-x_{1}, y_{1}, z_{1}$ and $o_{2}-x_{2}, y_{2}, z_{2}$ are static coordinate systems, and the other coordinate systems are moving coordinate systems. $o_{1}-x_{1}, y_{1}, z_{1}$ is connected with the cutter rotation axis, $o_{1}$ is located at the centre of the cutter. $o_{d 0}-x_{d 0}, y_{d 0}, z_{d 0}$ is also connected with the cutter rotation axis, and the location of coordinate origin $o_{d 0}$ is the same as of $o_{1}-x_{1}, y_{1}, z_{1}$, but it is a moving coordinate system. $o_{2}-x_{2}, y_{2}, z_{2}$ is connected with the blank of the CATT cylindrical gear, and $o_{2}$ is located at the centre of the gear blank. $o_{d}-x_{d}, y_{d}, z_{d}$ is also connected with the gear blank, and the location of coordinate origin $o_{d}$ is the same as of $o_{2}-x_{2}, y_{2}, z_{2}$, but it is a moving coordinate system. $R_{1}$ is the pitch circle of the gear blank; $\varphi$ is the rotation angle of the gear blank; $R_{\mathrm{T}}$ is the generative radius of the cutter (also called the tooth line radius); $\theta$ is the tooth profile position angle, which is from the cutter to the end face of the gear blank, and in the clockwise direction its value is positive; $B$ is the gear width of the gear blank. 


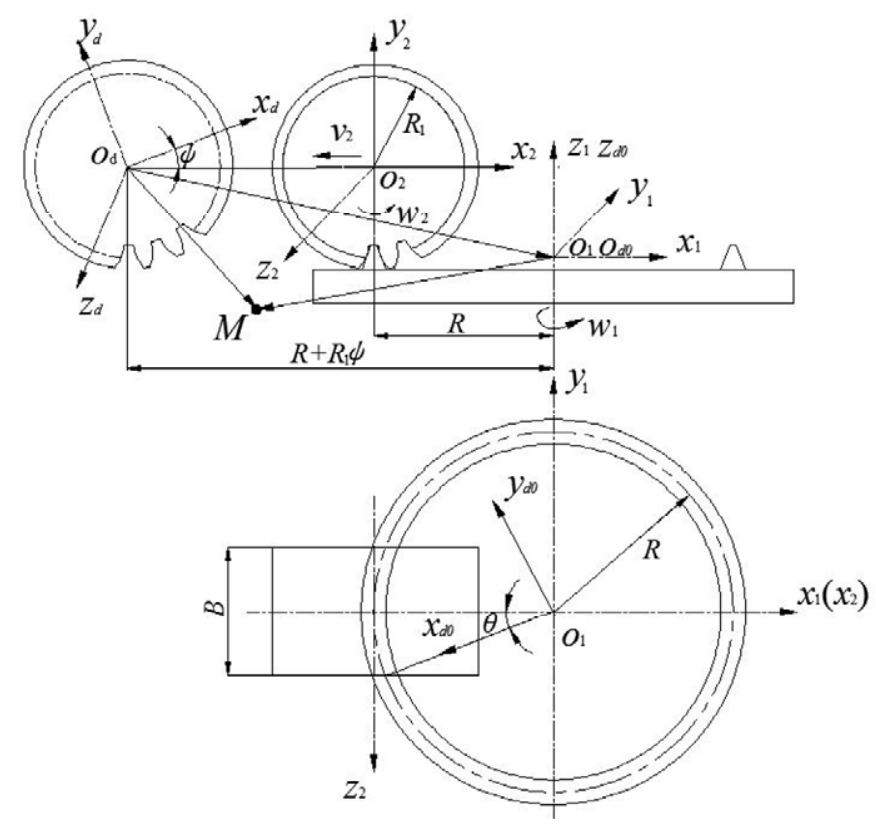

Fig. 1 Reference coordinate system of CATT cylindrical gear

In order to obtain the tooth surface equation of the CATT cylindrical gear, the coordinate system needs to be transformed. And the transformation process is as follows:

The coordinate transformation matrix $M_{1 d 0}$ from $o_{d 0}-x_{d 0}, y_{d 0}, z_{d 0}$ to $o_{1}-x_{1}, y_{1}, z_{1}$ can be written as:

$$
M_{1 d 0}=\left[\begin{array}{cccc}
\cos \theta & -\sin \theta & 0 & 0 \\
\sin \theta & \cos \theta & 0 & 0 \\
0 & 0 & 1 & 0 \\
0 & 0 & 0 & 1
\end{array}\right]
$$

The coordinate transformation matrix $M_{21}$ from $o_{1}-x_{1}, y_{1}, z_{1}$ to $o_{2}-x_{2}, y_{2}, z_{2}$ can be written as:

$$
M_{21}=\left[\begin{array}{cccc}
1 & 0 & 0 & R_{\mathrm{T}} \\
0 & 0 & 1 & -R_{1} \\
0 & -1 & 0 & 0 \\
0 & 0 & 0 & 1
\end{array}\right]
$$

The coordinate transformation matrix $M_{d 2}$ from $o_{2}-x_{2}, y_{2}, z_{2}$ to $o_{d}-x_{d}, y_{d}, z_{d}$ can be written as:

$$
M_{d 2}=\left[\begin{array}{cccc}
\cos \varphi & \sin \varphi & 0 & R_{1} \varphi \cos \varphi \\
-\sin \varphi & \cos \varphi & 0 & -R_{1} \varphi \sin \varphi \\
0 & 0 & 1 & 0 \\
0 & 0 & 0 & 1
\end{array}\right]
$$

So, the coordinate transformation from $o_{1}-x_{1}, y_{1}, z_{1}$ to $o_{d}-x_{d}, y_{d}, z_{d}$ can be expressed as:

$$
\left[\begin{array}{c}
x_{d} \\
y_{d} \\
z_{d} \\
1
\end{array}\right]=M_{d 2} \cdot M_{21} \cdot M_{1 d 0}\left[\begin{array}{c}
x_{d 0} \\
y_{d 0} \\
z_{d 0} \\
1
\end{array}\right]
$$

where $x_{d}, y_{d}, z_{d}$ are the coordinates of the gear tooth surface; $x_{d 0}, y_{d 0}, z_{d 0}$ are the coordinates of the cutter. 
To derive the tooth surface equation, the coordinate of the cutter in $o_{d 0}-x_{d 0}, y_{d 0}, z_{d 0}$ should be calculated first. The cutter blade geometry and parameters are shown in Fig. 2, where $h_{f}$ is the height of the gear root, and $h_{a}$ is the height of the addendum. The cutter equation in $o_{d 0}-x_{d 0}, y_{d 0}, z_{d 0}$ could be written as:

$$
\left\{\begin{array}{l}
x_{d 0}=-\left(R_{\mathrm{T}} \mp \frac{\pi}{4} m \pm u \sin \alpha\right) \\
y_{d 0}=0 \\
z_{d 0}=u \cos \alpha
\end{array}\right.
$$

where $m$ is the modulus of the gear width middle section, $\alpha$ is the pressure angle of the cutter, $u$ is the displacement from a random point on the cutting tool surface to a reference point in the direction of the cutting edge, ' $\mp$ ' represents the tool cutting edge inside and outside, respectively.

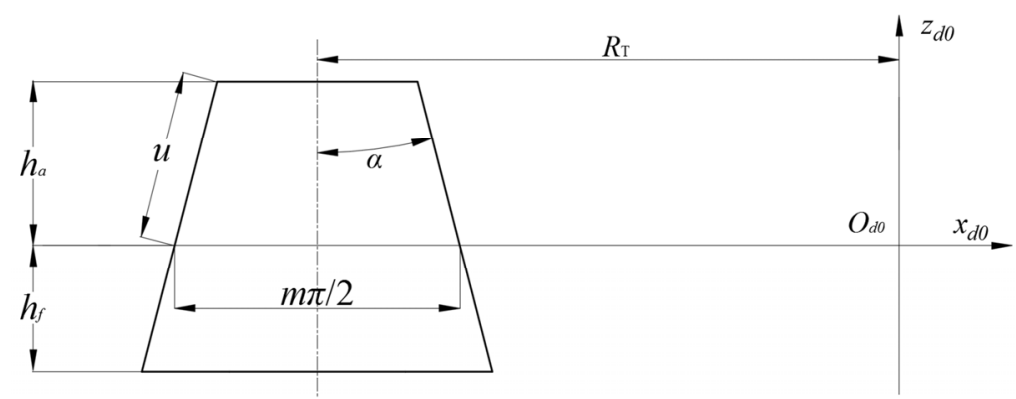

Fig. 2 Cutter blade geometry and parameters

Combining the space meshing condition [22] based on the meshing theory with Eq. (4) and Eq. (5), the tooth surface equation of the CATT cylindrical gear can be expressed as:

$$
\left\{\begin{aligned}
x_{d}= & {\left[-\left(R_{\mathrm{T}} \mp \frac{\pi}{4} m \pm u \sin \alpha\right) \cos \theta+R_{\mathrm{T}}+R_{1} \varphi\right] \cos \varphi } \\
& +\left(u \cos \alpha-R_{1}\right) \sin \varphi \\
y_{d}= & {\left[\left(R_{\mathrm{T}} \mp \frac{\pi}{4} m \pm u \sin \alpha\right) \cos \theta-R_{\mathrm{T}}-R_{1} \varphi\right] \sin \varphi } \\
& +\left(u \cos \alpha-R_{1}\right) \cos \varphi \\
z_{d}= & \left(R_{\mathrm{T}} \mp \frac{\pi}{4} m \pm u \sin \alpha\right) \sin \theta \\
u & =\mp \sin \alpha \frac{\cos \theta\left(R_{\mathrm{T}} \mp \frac{\pi}{4} m\right)-\left(R_{\mathrm{T}}+R_{1} \varphi\right)}{\cos \theta}
\end{aligned}\right.
$$

\section{Curvature and contact ellipse of the tooth surface}

\subsection{Normal curvature and main curvature}

To investigate the contact form of the CATT cylindrical gear, the normal curvature should be derived first. The method, by which the normal curvature is to be obtained, can be found in [23]. In the chapter "Tooth Surface Equation", the tooth surface equation of the CATT cylindrical gear has been derived; it could be rewritten as a vector expression, namely $\vec{r}$ :

$$
\vec{r}=x_{d} \vec{i}+y_{d} \vec{j}+z_{d} \vec{k}
$$

where $\vec{i}, \vec{j}$ and $\vec{k}$ are unit vectors in the direction of $x_{d}, y_{d}$, and $z_{d}$. 
The unit normal vector of tooth surface $\vec{n}$ is:

$$
\vec{n}=\frac{\vec{r}_{\theta} \times \vec{r}_{\varphi}}{\left|\vec{r}_{\theta} \cdot \vec{r}_{\varphi}\right|}
$$

where $\vec{r}_{\theta}, \vec{r}_{\varphi}$ are the first-order derivatives for $\theta$ and $\varphi$.

The first basic type of surface I is defined as:

$$
\begin{aligned}
\mathrm{I} & =\mathrm{d} \vec{r} \\
& =\left(\vec{r}_{\theta} \mathrm{d} \theta+\vec{r}_{\varphi} \mathrm{d} \varphi\right)^{2} \\
& =\vec{r}_{\theta}^{2} \mathrm{~d} \theta^{2}+2\left(\vec{r}_{\theta} \cdot \vec{r}_{\varphi}\right) \mathrm{d} \theta \mathrm{d} \varphi+\vec{r}_{\varphi}^{2} \mathrm{~d} \varphi^{2}
\end{aligned}
$$

where $\mathrm{d} \vec{r}, \mathrm{~d} \theta, \mathrm{d} \varphi$ are the first-order differentials for $\vec{r}, \theta, \varphi$.

And let:

$$
E=\vec{r}_{\theta}^{2}, F=\vec{r}_{\theta} \cdot \vec{r}, G=\vec{r}_{\varphi}^{2}
$$

The second basic type of surface II is defined as:

$$
\begin{aligned}
\mathrm{II}= & \mathrm{d}^{2} \vec{r} \cdot \vec{n} \\
& =\left(\vec{r}_{\theta \theta} \cdot \vec{n}\right) \mathrm{d} \theta^{2}+2\left(\vec{r}_{\theta \varphi} \cdot \vec{n}\right) \mathrm{d} \theta \mathrm{d} \varphi+ \\
& \left(\vec{r}_{\varphi \varphi} \cdot \vec{n}\right) \mathrm{d} \varphi^{2}+\left(\vec{r}_{\theta} \cdot \vec{n}\right) \mathrm{d}^{2} \theta+\left(\vec{r}_{\varphi} \cdot \vec{n}\right) \mathrm{d}^{2} \varphi
\end{aligned}
$$

where $\mathrm{d} \varphi^{2}, \mathrm{~d} \theta^{2}$ are the second-order differentials for $\varphi, \theta ; \vec{r}_{\theta \theta} \vec{r}_{\varphi \varphi} \vec{r}_{\theta \varphi}$ are the second-order derivates for $\theta, \varphi, \varphi$ and $\theta$.

And let:

$$
L=\vec{r}_{\theta \theta} \cdot \vec{n}, \quad M=\vec{r}_{\theta \varphi} \cdot \vec{n}, \quad N=\vec{r}_{\varphi \varphi} \cdot \vec{n}
$$

Surface Gaussian curvature $K$ and average curvature $H$ are expressed as:

$$
\begin{gathered}
K=\frac{L N-M^{2}}{E G-F^{2}} \\
H=\frac{L G-2 M F+N E}{2\left(E G-F^{2}\right)}
\end{gathered}
$$

The main curvatures $k_{1}$ and $k_{2}$ satisfy Eq. (15):

$$
x^{2}-H x+K=0
$$

Namely, the roots of Eq. (15) are the main curvatures $k_{1}$ and $k_{2}$. So the main curvatures $k_{1}$ and $k_{2}$ can be expressed as:

$$
k_{1}=H+\sqrt{H^{2}-K}, \quad k_{2}=H+\sqrt{H^{2}+K}
$$

Because the direction has an effect on the normal curvature, in order to study the surface characteristics accurately, the main curvature was regarded as the research target. As for the gear surface, the gear's geometrical characteristics depend on the geometric parameters in the direction of the tooth profile and tooth line. Therefore, one only needs to focus on the main curvature in the direction of the tooth profile and tooth line.

\subsection{Induced normal curvature}

In the meshing process, the gear contact is a result of interaction between two gear tooth surfaces, so it is not enough just to analyze one gear's normal curvature, it is necessary to study the relative normal curvature of the tooth surfaces, namely, the induced normal curvature. 
Assuming that $\Sigma^{\mathrm{I}}$ is the concave surface of the CATT cylindrical gear, $\Sigma^{\mathrm{II}}$ is the convex surface of the CATT cylindrical gear. $\Sigma^{\mathrm{I}}$ is tangent to $\Sigma^{\mathrm{II}}$ at point $M$. The main directions of $\Sigma^{\mathrm{I}}$ are $e_{1}^{\mathrm{I}}$ and $e_{2}^{\mathrm{I}}$, and the main curvatures are $k_{1}^{\mathrm{I}}$ and $k_{2}^{\mathrm{I}}$. The main directions of $\sum^{\mathrm{II}}$ are $e_{1}^{\mathrm{II}}$ and $e_{2}^{\mathrm{II}}$, and the main curvatures are $k_{1}^{\mathrm{II}}$ and $k_{2}^{\mathrm{II}} \cdot \sigma$ is the directed angle from $e_{1}^{\mathrm{I}}$ to $e_{1}^{\mathrm{II}}$. And the concave and convex surfaces have the same normal vector at point $M$. The curvature relationship of the tangent surfaces is shown in Fig. 3.

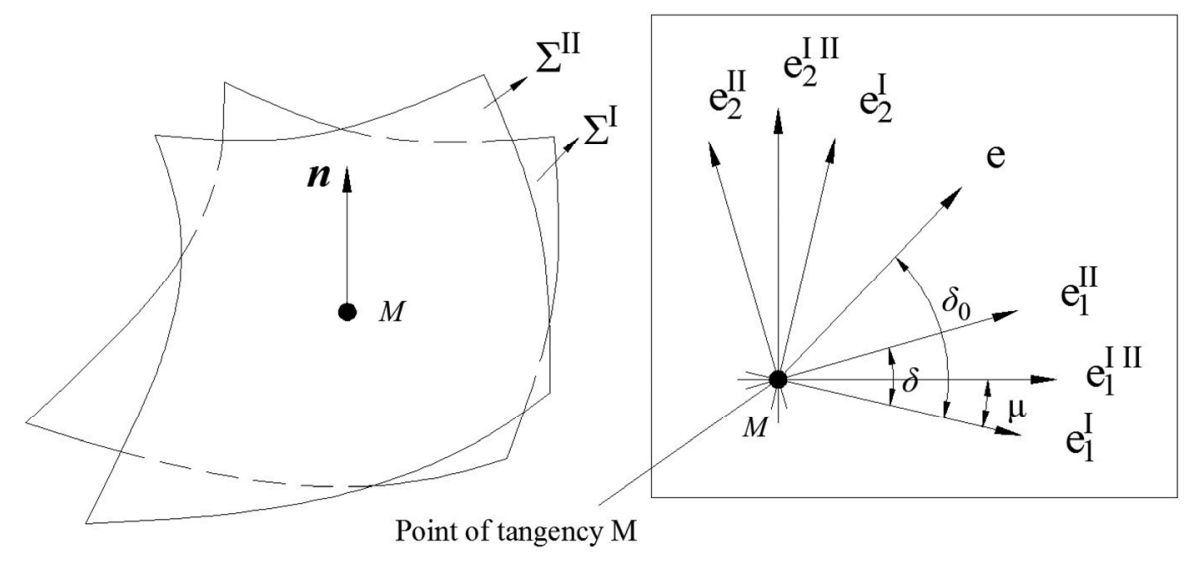

(a) Tangent surfaces

(b) Curvature relationship

Fig. 3 Curvature relationship of tangent surfaces

In the arbitrary direction $e$, in which the directed angle is $\sigma_{0}$ from $e_{1}^{\mathrm{I}}$ to $e$, the normal curvature of $\sum^{\mathrm{I}}$ is expressed as:

$$
\begin{aligned}
k^{\mathrm{I}} & =k_{1}^{\mathrm{I}} \cos ^{2} \sigma_{0}+k_{2}^{\mathrm{I}} \sin ^{2} \sigma_{0} \\
& =H^{\mathrm{I}}+R^{\mathrm{I}} \cos 2 \sigma_{0}
\end{aligned}
$$

where $R^{\mathrm{I}}=\frac{1}{2}\left(k_{1}^{\mathrm{I}}-k_{2}^{\mathrm{I}}\right)$.

In the same direction, the normal curvature of $\sum^{\mathrm{II}}$ is expressed as:

$$
\begin{aligned}
k^{\mathrm{II}} & =k_{1}^{\mathrm{II}} \cos ^{2}\left(\sigma_{0}-\sigma\right)+k_{2}^{\mathrm{II}} \sin ^{2}\left(\sigma_{0}-\sigma\right) \\
& =H^{\mathrm{II}}+R^{\mathrm{II}} \cos 2\left(\sigma_{0}-\sigma\right)
\end{aligned}
$$

where $R^{\mathrm{II}}=\frac{1}{2}\left(k_{1}^{\mathrm{II}}-k_{2}^{\mathrm{II}}\right)$.

In the same direction, the difference between the normal curvatures of the two tooth surfaces at the tangent point is expressed as:

$$
\begin{aligned}
k^{\mathrm{I}} \mathrm{II} & =k^{\mathrm{I}}-k^{\mathrm{II}} \\
& =H^{\mathrm{I}}-H^{\mathrm{II}}+R^{\mathrm{II}} \cos 2 \sigma_{0}-R^{\mathrm{II}} \cos 2\left(\sigma_{0}-\sigma\right)
\end{aligned}
$$

Namely, Eq. (19) is the induced normal curvature between the concave and convex surfaces. According to [23], two main values of the induced normal curvature are expressed as:

$$
\left\{\begin{array}{l}
k_{1}^{\text {I II }}=k_{1}^{\mathrm{I}}-k_{1}^{\mathrm{II}} \\
k_{2}^{\mathrm{I}}{ }^{\mathrm{II}}=k_{2}^{\mathrm{I}}-k_{2}^{\mathrm{II}}
\end{array}\right.
$$

\subsection{Contact ellipse}

When the tooth surfaces are loaded, if the contact area of the gear with a point contact is projected onto the tangency plane of the meshing point, the contact area generally appears as an ellipse, as shown in Fig. 4. Also, the centre of the instantaneous contact ellipse overlaps with the theoretical contact point. Besides, the elastic deformation $\delta$ of the gear surface 
depends on the load, but it is generally considered as a fixed value. In this paper, according to [23], this study mainly aimed at the light load, so let the elastic deformation $\delta$ be equal to $0.00632 \mathrm{~mm}$.

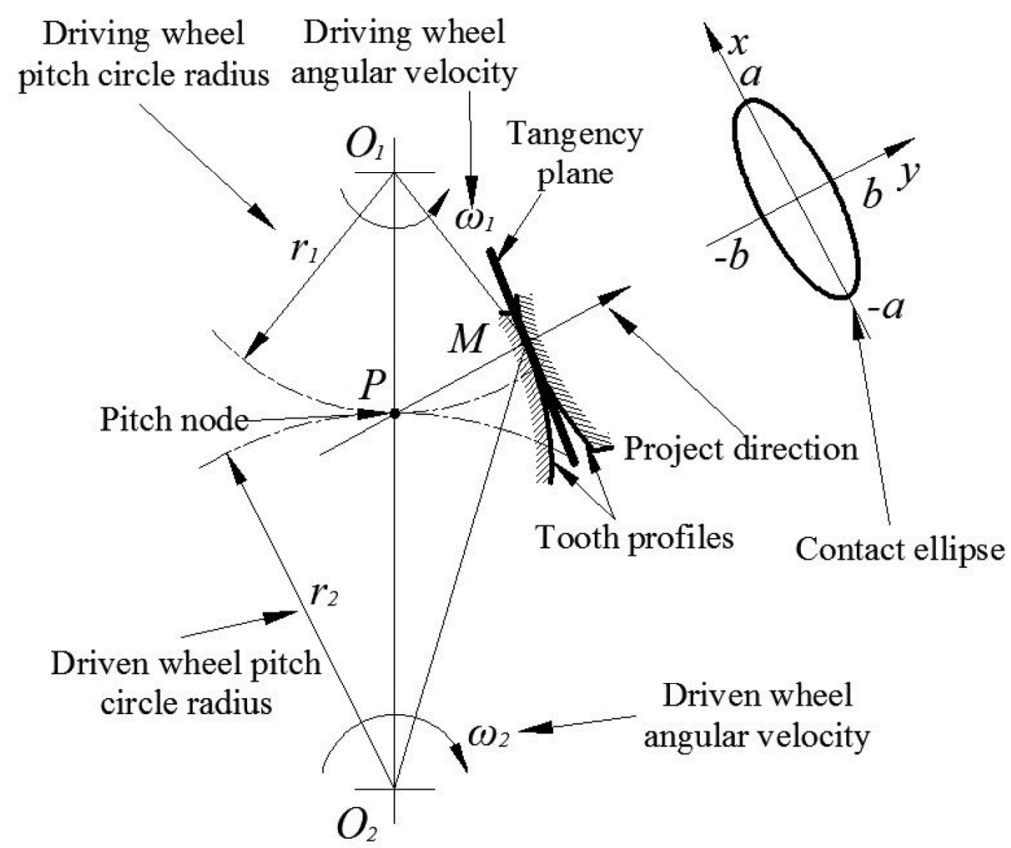

Fig. 4 Contact ellipse

According to the above analysis, the contact ellipse equation of the CATT cylindrical gear is defined as follows:

$$
A \xi^{2}+B \eta^{2}= \pm \delta
$$

Long axis $a$ and short axis $b$ of the contact ellipse can be expressed as:

$$
\begin{gathered}
a=\left|\frac{\delta}{A}\right|^{1 / 2}, b=\left|\frac{\delta}{B}\right|^{1 / 2} \\
A=\frac{1}{4}\left[k_{\Sigma}^{\mathrm{I}}-k_{\Sigma}^{\mathrm{II}}-\left(g_{\mathrm{I}}^{2}-2 g_{\mathrm{I}} g_{\mathrm{II}} \cos 2 \sigma+g_{\mathrm{II}}^{2}\right)^{1 / 2}\right] \\
B=\frac{1}{4}\left[k_{\Sigma}^{\mathrm{I}}-k_{\Sigma}^{\mathrm{II}}+\left(g_{\mathrm{I}}^{2}-2 g_{\mathrm{I}} g_{\mathrm{II}} \cos 2 \sigma+g_{\mathrm{II}}^{2}\right)^{1 / 2}\right] \\
k_{\Sigma}^{i}=k_{1}^{i}+k_{2}^{i}(i=\mathrm{I}, \mathrm{II}) g_{i}=k_{1}^{i}-k_{2}^{i}(i=\mathrm{I}, \mathrm{II}) \\
\cos 2 \alpha^{\mathrm{I}}=\frac{g_{\mathrm{I}}-g_{\mathrm{II}} \cos 2 \sigma}{\left(g_{\mathrm{I}}^{2}-2 g_{\mathrm{I}} g_{\mathrm{II}} \cos 2 \sigma+g_{\mathrm{II}}^{2}\right)^{1 / 2}}
\end{gathered}
$$

The elliptical area can be represented as:

$$
S=\pi a b
$$

According to the above deduction, in theory, the contact of the CATT cylindrical gear is point contact. Because of the elasticity deformation under the load, the contact area becomes an ellipse. Also, the centre of the instantaneous contact ellipse overlaps with the theoretical contact point. In fact, in the entire process of gear meshing, the contact trace is a series of contact ellipses.

\section{Validation of the contact form of CATT cylindrical gear}

In order to prove the contact form of the CATT cylindrical gear, an accurate 3D model was developed by using MATLAB and UG NX 8.0. The gear parameters are as follows: 
number of teeth $z_{1}=19$ and $z_{2}=31$, gear modulus $m=8 \mathrm{~mm}$, gear width $B=80 \mathrm{~mm}$, tooth line radius $R_{\mathrm{T}}=500 \mathrm{~mm}$ and the rest of the parameters are selected according to the standard [24], for example, pitch circle pressure angle $\alpha=20^{\circ}$, addendum coefficient $h^{*}=1$ and coefficient of bottom clearance $c^{*}=0.25$. To set up the model, the tooth pastry cloud was obtained from MATLAB first, and then an accurate 3D model was developed and assembled in UG NX 8.0, as shown in Fig. 5 and Fig. 6.

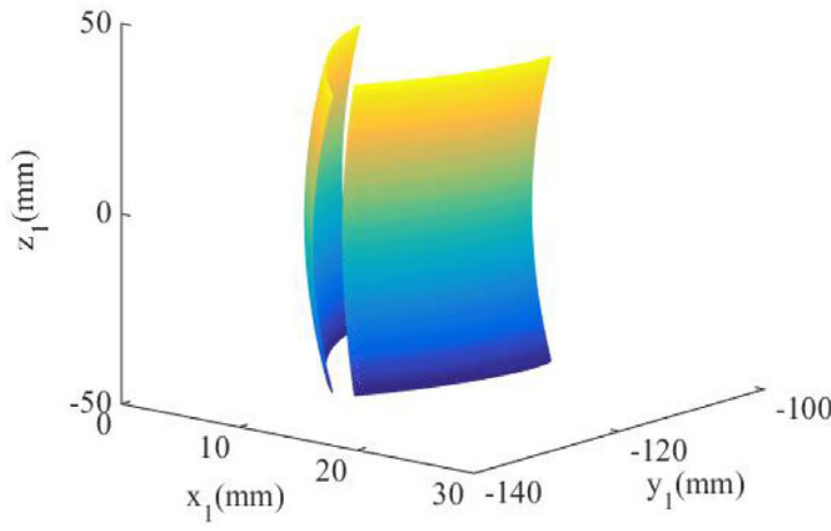

Fig. 5 Tooth pastry cloud

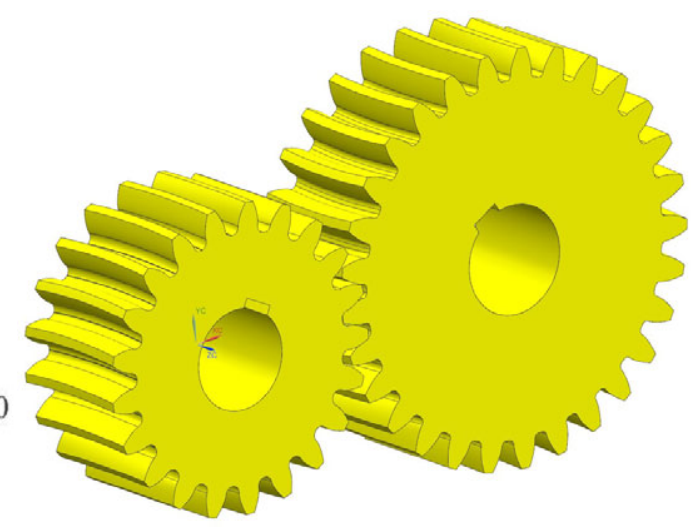

Fig. 6 Accurate assembly

The assembly model was imported into ANSYS Workbench. The application of the finite element method requires the development of finite element models which are formed of a finite element mesh, the definition of contacting surfaces, the establishment of boundary conditions and the loading of the gear drive with a desired torque [25]. The important parameters and the boundary conditions are set as follows: medium carbon steel (AISI 1045) was selected as the material for the FEA model, and Young's modulus and Poisson's ratio of this material are $210 \mathrm{GPa}$ and 0.29 , respectively. The sliding and static friction coefficients of the contacting surfaces are equal to 0.05 and 0.15 , respectively. All degrees of freedom of the driven wheel were fully fixed, the driving wheel retained only the rotational degrees of freedom of the $\mathrm{z}$ axis, and the rest were fixed. Moment load $M=3000 \mathrm{~N} \cdot \mathrm{m}$ was applied to the driving wheel. The von-Mises stress was set as a solution output. A static finite element analysis model of the CATT cylindrical gear is presented in Fig. 7.

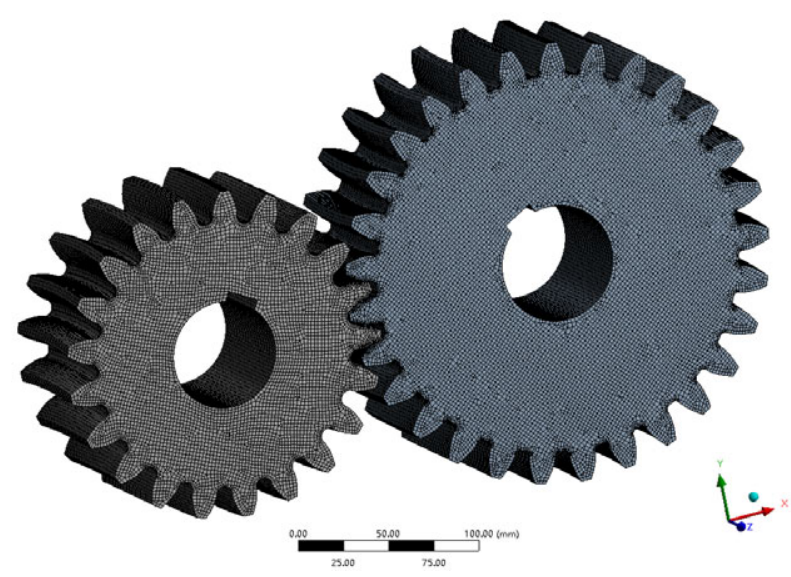

Fig. 7 Finite element analysis model of explicit dynamics

In the simulation, the static contact stress cloud pictures were obtained as shown in Fig. 8. According to the meshing features of the gear, the maximal contact stress usually appears in the contact area because of the positive pressure and it appears on both sides of the gear tooth root because of the bending stress. According to the stress cloud pictures of the 
CATT cylindrical gears, the maximal contact stress of both the driving and the driven gear appears in the contact area which has a form of approximate ellipses. Namely, the form of the CATT cylindrical gear's contact is the point contact.

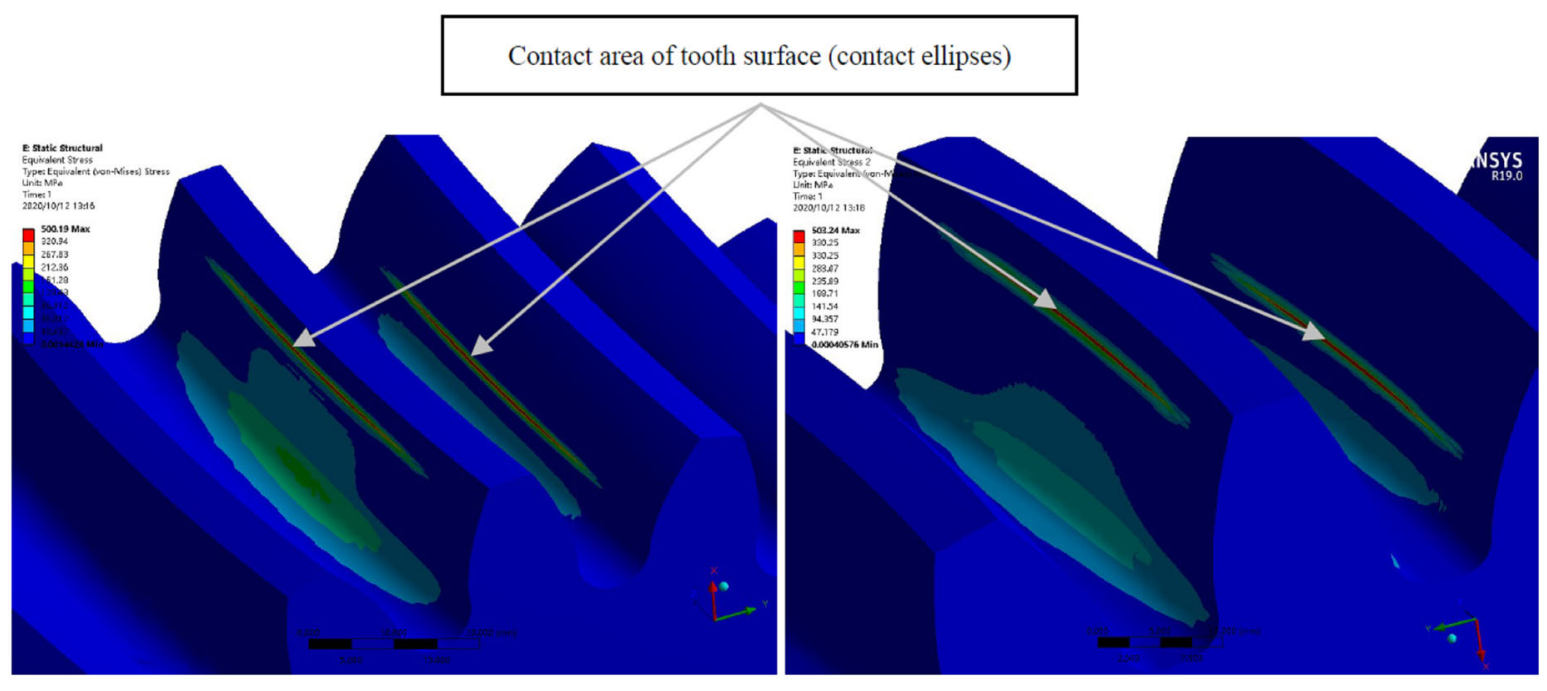

(a) Driving gear

(b) Driven gear

Fig. 8 Static contact stress in driving and driven gears of CATT cylindrical gear pair

To prove the contact form of the CATT cylindrical gear additionally, the gears have been cut by a numerical control machine, and the processed gears are installed in the CATT cylindrical gear reducer. At the same time, vermilion was applied to some tooth surfaces of the driving and the driven gear tooth, as shown in Fig. 9, and it is obvious that vermilion is homogeneous across the tooth surfaces. The gear parameters are as follows: number of teeth $z_{1}=31$ and $z_{2}=69$, gear modulus $m=4 \mathrm{~mm}$, driving gear width $B=85 \mathrm{~mm}$, driven gear width $B=80 \mathrm{~mm}$, tooth line radius $R_{\mathrm{T}}=500 \mathrm{~mm}$, and the rest of the parameters are also selected according to the standard [24].

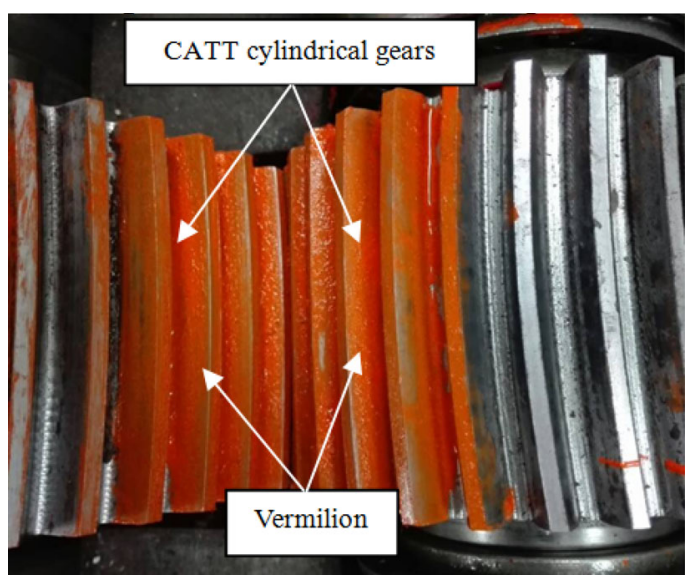

Fig. 9 Vermilion applied to gear surfaces

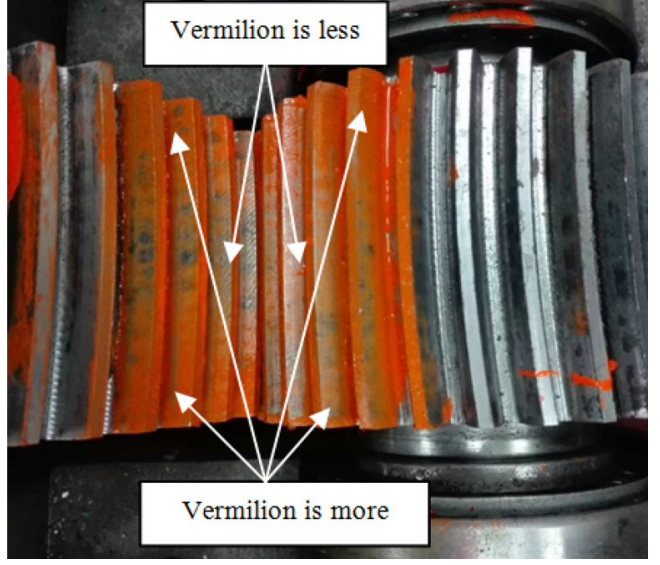

Fig. 10 Meshing mark on tooth surfaces

The test teeth were rotating several times and vermilion was observed across the tooth surfaces. It is obvious that the least vermilion was found in the middle cross section of the tooth surfaces. And the closer to the end face, the more vermilion on the tooth surfaces can be seen, as shown in Fig. 10. The reason for this is the following: on the one hand, there is mutual contact and meshing motion between the tooth surfaces; on the other hand, the tooth thickness becomes thin gradually beginning from the middle cross section to the end surface; there may be no contact at the end face, that is to say, the contact area is found mainly in the middle section of the tooth width, which phenomenon has also been observed in [26]. In fact, 
the whole contact area of the tooth surface consists of many continuous contact elastic deformation areas in the finite region of symmetry around the middle section, and the contact elastic deformation area is a contact ellipse, as shown in Fig.11. Namely, the form of the contact of the CATT cylindrical gear is the point contact.

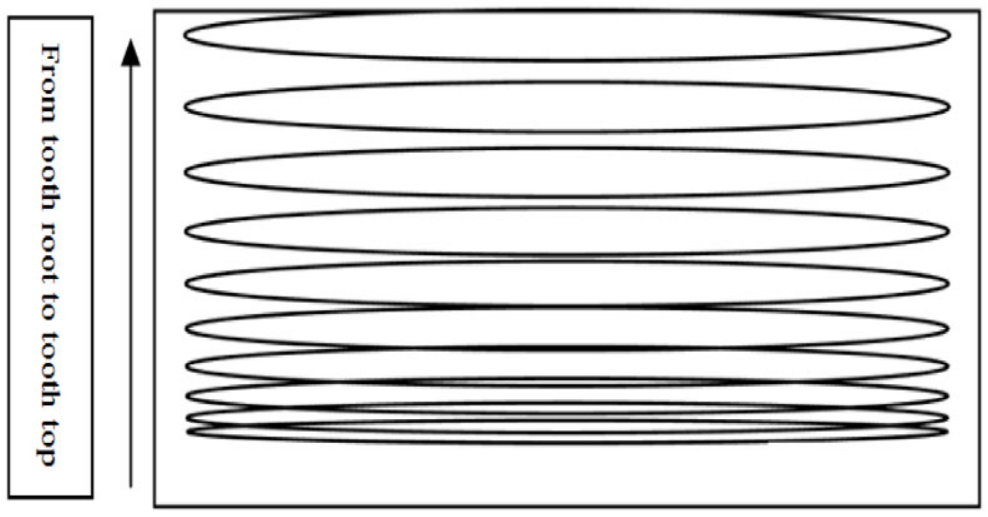

Fig. 11 Contact ellipses at different meshing points

\section{Influences of design parameters on contact ellipse}

The CATT cylindrical gear is a new gear transmission. It has characteristics of traditional gear transmissions. The tooth line along the tooth width is a spatial arc, the tooth profile of the CATT cylindrical gear is involute in the middle cross section, and the tooth profile of the other arbitrary cross section is hyperbolic. It can overcome disadvantages of spur gears, helical gears and herringbone gears; for example, spur gears generate shock and noise due to entering or exiting meshing at the same time, a helical gear transmission has a complicated shafting structure due to the axial force, and herringbone gears have complicated processing techniques and cause unbalanced loads easily. The main structural parameters of gear teeth include modulus, teeth number, pressure angle and tooth line radius. The parameters have some influence on the contact area of the CATT cylindrical gear, and then the parameters affect the transmission performance of the gear system further.

Figs. 12-15 show the influence of the modulus, gear tooth numbers and tooth line radius on the elliptical contact area. In the figures, the elliptical contact area is the projection of the actual contact area on the tangent surface of the meshing point, and it is calculated according to formula given in Eq. (27). The horizontal axis is the gear rotation angle, the value of $\varphi_{1}$ ranges from $-14.5^{\circ}$ to $14.5^{\circ}$, and it indicates that the contact position changes from the gear root to the gear top, and the gear rotation angle at the pitch node is equal to zero.

\subsection{Influence of modulus on the elliptical contact area}

The modulus is an important parameter of gear design, and it has an important influence on the geometric properties of the tooth surface and contact performances of the CATT cylindrical gear. Fig. 12 shows the influence of the modulus on the elliptical contact area. The parameters are as follows: $\mathrm{z}_{1}=21 ; \mathrm{z}_{2}=29 ; R_{\mathrm{T}}=500 \mathrm{~mm} ; B=80 \mathrm{~mm} ; m=4 \mathrm{~mm}, 8 \mathrm{~mm}, 12 \mathrm{~mm}$, and $16 \mathrm{~mm}$, respectively.

It is obvious from the graphs that the gear width has an effect on the elliptical contact area. On the whole, this area increases gradually from the gear root to the tooth top regardless of the modulus. However, the modulus has different effects on the elliptical contact area at different meshing points, as shown in the magnified rectangular area in Fig. 12. When the meshing point is near the tooth root, the elliptical contact area decreases when the modulus increases; when the meshing point is near the tooth top, the elliptical contact area increases when the modulus increases. And it is obvious that the elliptical contact area decreases in a 
cliff-like manner near the tooth top. Because the real and effective contact section of the tooth surfaces is a limited area, the contact ellipse near the gear top will extend beyond this limited area. That is to say, in a real situation, there is no contact in the part of the contact ellipse, namely, the contact section area will decrease.

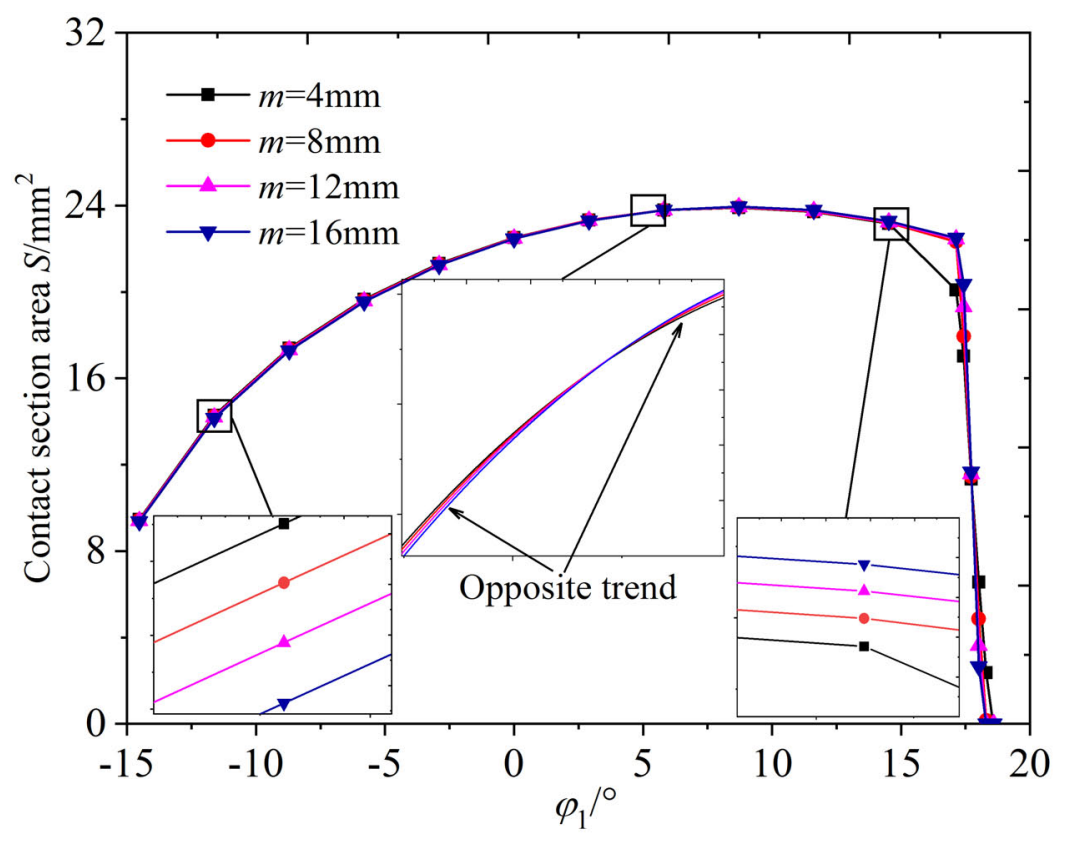

Fig. 12 Influence of modulus on elliptical contact area

\subsection{Influence of tooth line radius on elliptical contact area}

The tooth line radius is also an important parameter of the CATT cylindrical gear, and it is the main structural feature that is different from spur gears, helical gears and herringbone gears, and it also has an important influence on the elliptical contact area of CATT cylindrical gears. Fig. 13 shows the influence of the tooth line radius on the elliptical contact area. The parameters are as follows: $z_{1}=21 ; z_{2}=29$ and $89 ; B=80 \mathrm{~mm} ; m=8 \mathrm{~mm} ; R_{\mathrm{T}}=300 \mathrm{~mm}, 400 \mathrm{~mm}$, $500 \mathrm{~mm}, 800 \mathrm{~mm}$, and $600 \mathrm{~mm}$, respectively.
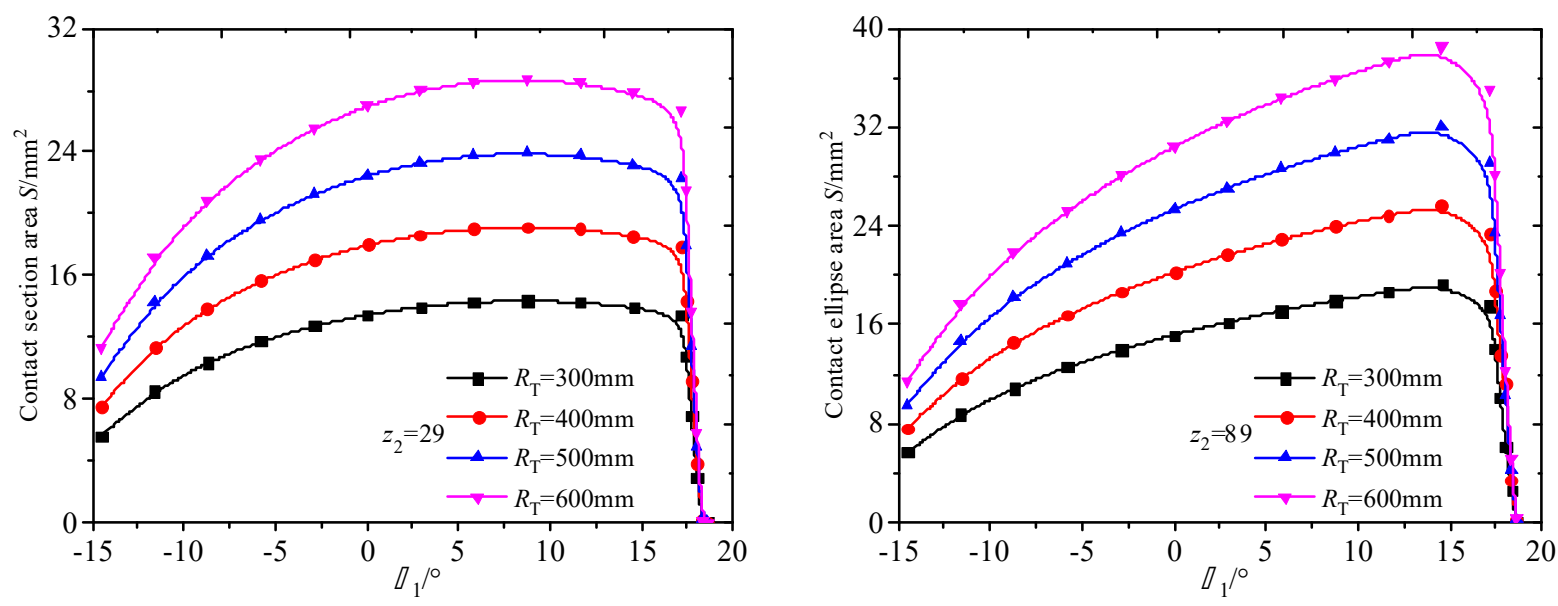

Fig. 13 Influence of tooth line radius on elliptical contact area

From the graphs, it is obvious that the tooth line radius affects the elliptical contact area. On the whole, the elliptical contact area increases gradually from the gear root to the top of the tooth regardless of the tooth line radius, however, the rate of the increase in the elliptical contact area decreases gradually from the gear root to the top of the tooth. At the same 
meshing point of the tooth surface, the elliptical contact area increases when the tooth line radius increases, however, the rate of the increase in the elliptical contact area decreases gradually with an increase in the tooth radius. And it is also obvious that the elliptical contact area decreases in a cliff-like manner near the tooth top.

The reason for the increase in the elliptical contact area is the following: the profile of the CATT cylindrical gear is involute in the middle cross section, the tooth profile of another arbitrary cross section is hyperbolic, and the tooth thickness would change with a change in the tooth line radius. According to [26], the tooth profile equation of the arbitrary cross section can be represented as:

$$
\left\{\begin{array}{l}
x_{1}=\left[-\left( \pm u \sin \alpha+R_{T} \pm \frac{\pi m}{4}\right) \cos \theta+R_{T}+R_{1} \varphi_{1}\right] \cos \varphi_{1}-\left(R_{1}+u \cos \alpha\right) \sin \varphi_{1} \\
y_{1}=\left[-\left( \pm u \sin \alpha+R_{T} \pm \frac{\pi m}{4}\right) \cos \theta+R_{T}+R_{1} \varphi_{1}\right] \sin \varphi_{1}+\left(R_{1}+u \cos \alpha\right) \cos \varphi_{1} \\
\varphi_{1}=-\frac{1}{R_{1}}\left\{\left[\frac{b}{\sin \theta}+\cos ^{2} \alpha\left(R_{T} \pm \frac{m \pi}{4}\right)\right] \frac{\cos \theta}{\sin ^{2} \alpha}+R_{T}\right\} \\
u=\mp \frac{\sin \alpha}{\cos \theta}\left[\cos \theta\left(R_{T} \pm \frac{\pi m}{4}\right)-\left(R_{T}+R_{1} \varphi_{1}\right)\right]
\end{array}\right.
$$

In the middle cross section, the tooth profile equation can be represented as:

$$
\left\{\begin{array}{l}
x_{1}=\left(R_{1} \varphi_{1} \cos \alpha \mp \frac{\pi m}{4} \cos \alpha \pm R_{1} \sin \alpha\right) \cos \left(\varphi_{1} \pm \alpha\right)-R_{1} \sin \left(\varphi_{1} \pm \alpha\right) \cos \alpha \\
y_{1}=\left(R_{1} \varphi_{1} \cos \alpha \mp \frac{\pi m}{4} \cos \alpha \pm R_{1} \sin \alpha\right) \sin \left(\varphi_{1} \pm \alpha\right)+R_{1} \cos \left(\varphi_{1} \pm \alpha\right) \cos \alpha
\end{array}\right.
$$

Based on the tooth profile equation, the influence of the tooth line radius on the tooth thickness is shown in Fig. 14. In the graphs, B0 expresses the middle cross section; B15, B30 and $\mathrm{B} 45$ indicate that the distance from the measured cross section to the middle cross section is $15 \mathrm{~mm}, 30 \mathrm{~mm}$, and $45 \mathrm{~mm}$, respectively. From the graphs, it is obvious that the tooth line radius has almost no influence on the tooth thickness in the middle cross section; the tooth thickness becomes thinner and thinner when the tooth line radius in other arbitrary cross section decreases in the direction of the gear width. That is to say, the space between the concave and the convex tooth surfaces increases when the tooth line radius decreases in the direction of the gear width, and this makes the contact area become smaller and smaller. At the same time, when the radius of the tooth surface is too large, the change in the tooth thickness is small, so the change in the contact area is also small.

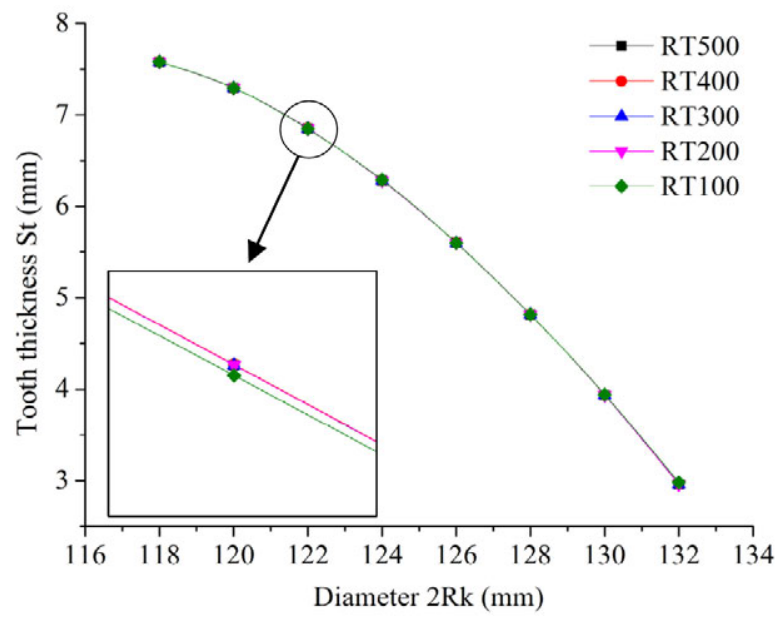

a) $\mathrm{B} 0$

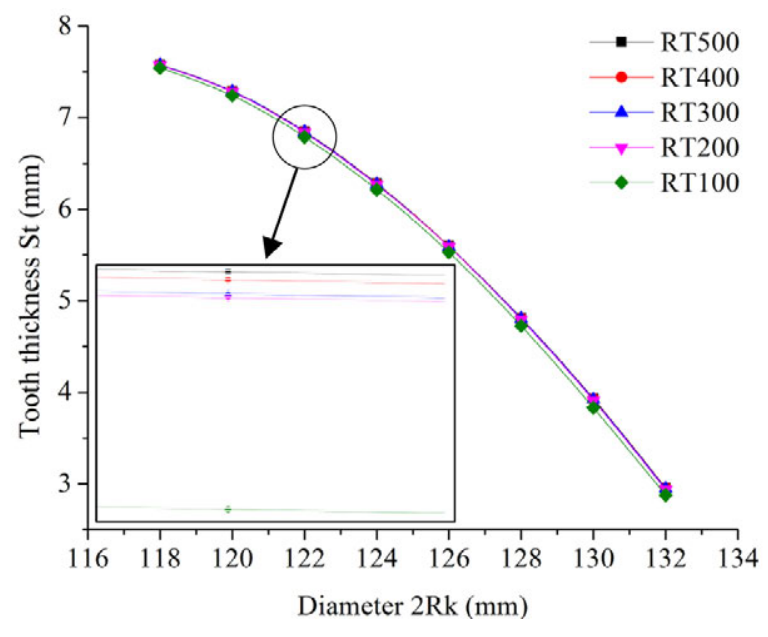

b) B15 


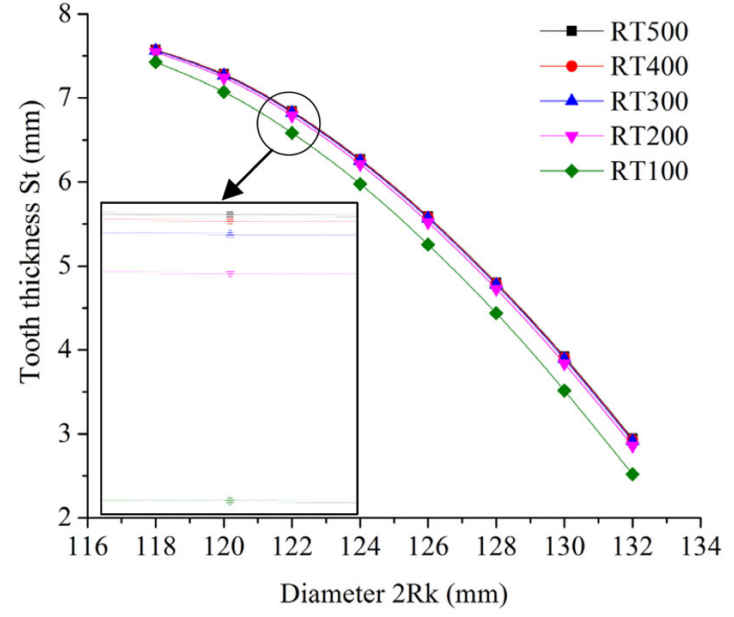

c) B30

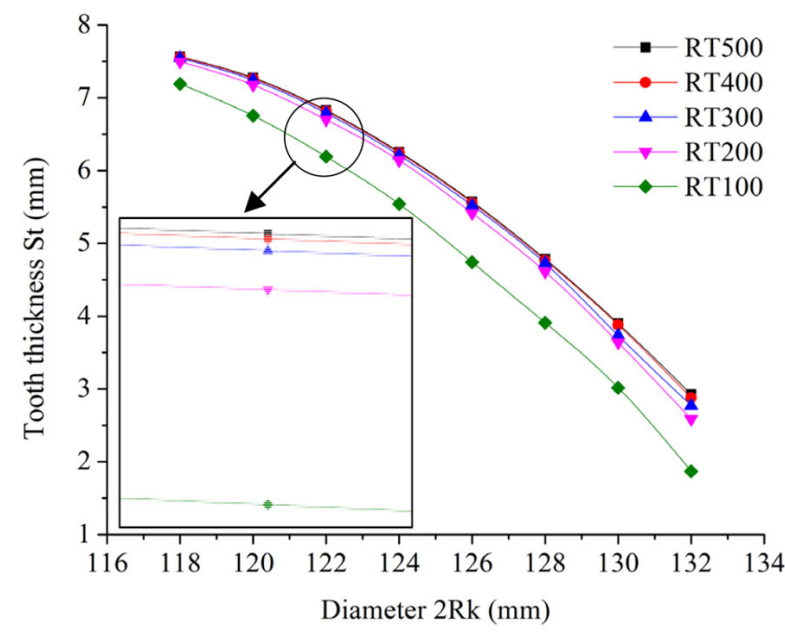

d) B45

Fig. 14 Influence of tooth line radius on tooth thickness

\subsection{Influence of gear tooth numbers on elliptical contact area}

The gear tooth number is also an important parameter of CATT cylindrical gears, it determines the size of the gear, and it also has an important influence on the contact area of the CATT cylindrical gear. Fig. 15 shows the influence of the gear tooth number on the elliptical contact area. The parameters are as follows: $\mathrm{z}_{1}=21 ; m=8 \mathrm{~mm} ; R_{\mathrm{T}}=500 \mathrm{~mm} ; B=80 \mathrm{~mm}$; $\mathrm{z}_{2}=29,49,69,89$, and 109, respectively.

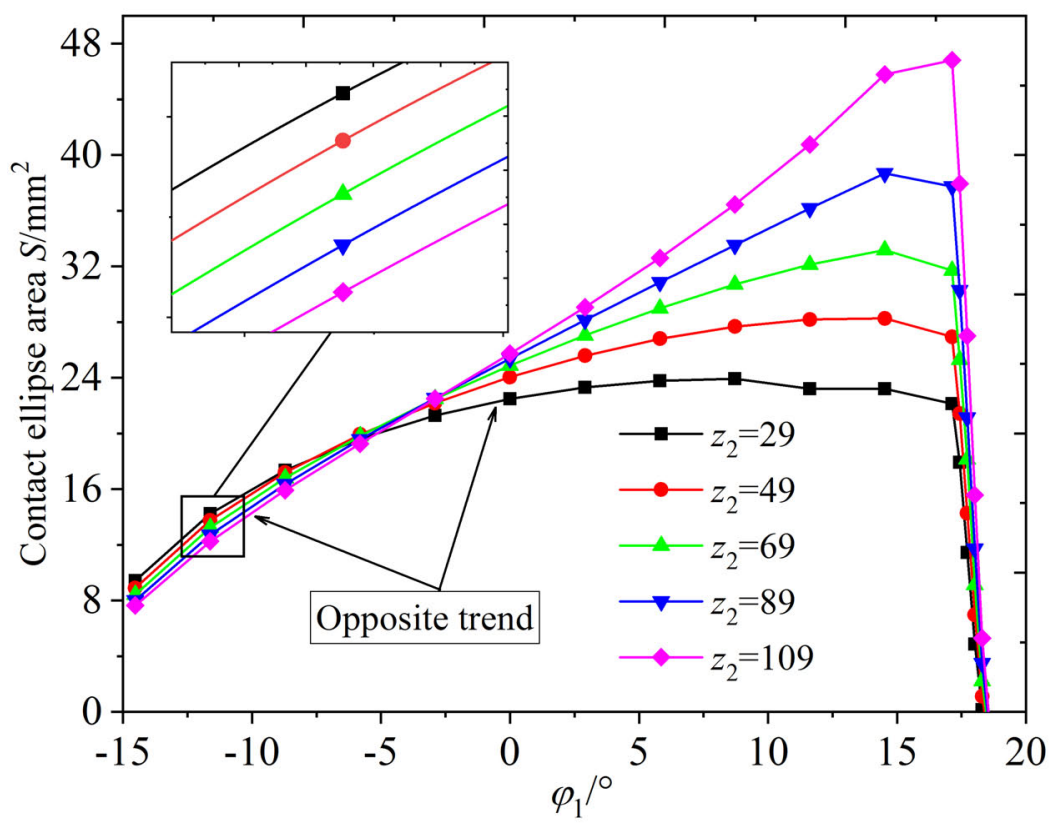

Fig. 15 Influence of gear tooth number on elliptical contact area

From the graphs, the effect of the gear tooth number on the elliptical contact area is obvious. On the whole, the elliptical contact area increases gradually from the gear root to the tooth top regardless of the gear tooth number, and the rate of the increase in the elliptical contact area decreases gradually from the gear root to the tooth top. However, the gear tooth number has different effects on the elliptical contact area at different meshing points, as shown in the magnified rectangular area in Fig. 15. When the meshing point is near the tooth root, the elliptical contact area decreases when the gear tooth number increases. When the 
meshing point is near the tooth top, the elliptical contact area increases when the gear tooth number increases. The position near the top of the tooth is more affected by the number of teeth, and the point not affected by the number of teeth is near the tooth root. And it is also obvious that the change in the elliptical contact area near the tooth top is the same as the influence of the tooth line radius and modulus.

\section{Conclusion}

This paper discusses the tooth surface reconstruction and characteristics of the surface contact in CATT gears. Firstly, based on the meshing theory, the tooth surface equation, tooth surface curvature equation and tooth surface contact ellipse equation of the CATT cylindrical gear were deduced to prove the contact form of the CATT cylindrical gear. Next, the tooth surface was reconstructed and a 3D model was developed. Then, the finite element analysis and meshing impression experiment were used to prove the contact form additionally. Finally, the influences of the design parameters on the contact ellipse were investigated. The main conclusions can be expressed as follows:

(1) The gear surface equation, the induced normal curvature equations and contact ellipse of the CATT cylindrical gear were derived. It is proved that the contact is a point contact. When the tooth surfaces are loaded, if the contact area is projected onto the tangent plane of the meshing point, the contact area generally appears as an ellipse.

(2) A 3D model of the CATT cylindrical gear was developed. The finite element analysis and meshing impression experiment were used to prove the contact form additionally. Observing the experimental results, the maximum stress region of the finite element model appears as an approximate ellipse at the contact position and the least vermilion was found in the central region of the tooth surfaces. It indicates that the contact form of the CATT cylindrical gear pair is the point contact.

(3) The results of the analysis showed that the general tendency is that the elliptical contact area of the tooth surfaces increases from the tooth root towards the gear top; the elliptical contact area decreases when the modulus and the gear tooth number near the tooth root increase; the elliptical contact area increases when the modulus and the gear tooth number near the tooth top increase; the elliptical contact area increases when the tooth line radius increases, and the rate of speed in the elliptical contact area decreases when the tooth radius increases. In addition, the elliptical contact area decreases in a cliff-like manner near the tooth top.

\section{Acknowledgement}

This study was funded by the Guizhou Province Education Department Young Science and Technology Talents Growth Project (grant number [2018] 319), the Zunyi Science and Technology Bureau, the Zunyi Normal University Joint Science and Technology Fund Project (grant number [2018] 13), the Guizhou Provincial Natural Science Foundation (grant number [2018]5784-03) and Guizhou Provincial Natural Science Foundation (grant number [2017]1203).

\section{Reference}

[1] Ding H, Tang J, Zhong J, et al. A hybrid modification approach of machine-tool setting considering high tooth contact performance in spiral bevel and hypoid gears. Journal of Manufacturing Systems, 2016, 41, 228-238. https://doi.org/10.1016/j.jmsy.2016.09.003 
[2] Simon V V. Improvements in the mixed elastohydrodynamic lubrication and in the efficiency of hypoid gears. Proceedings of the Institution of Mechanical Engineers Part J Journal of Engineering Tribology, 2019, 208-210, 1-16. https://doi.org/10.1177/1350650119866027

[3] Simon V V. Multi-objective optimization of hypoid gears to improve operating characteristics. Mechanism and Machine Theory, 2020, 146,103727. https://doi.org/10.1016/j.mechmachtheory.2019.103727

[4] Aiping S. Involute arc cylindrical gear and its mesh characteristics. China Mechanical Engineering 2006, 17(18), 1888-1892.

[5] Di Y., Chen M. Generation principle of accurate tooth traces cylindrical gear. Journal of Harbin Bearing. 2006, 38(3), 58-61.

[6] Zhang, Q., Hou, L., Tang, R. Method of Processing and an Analysis of Meshing and Contact of Circular Arc Tooth Trace Cylindrical Gears. Transactions of FAMENA, 2016, 40(4), 11-24. https://doi.org/10.21278/tof.40402

[7] F.L. Litvin, A.M. Egelja, M. De Donno. Computerized determination of singularities and envelopes to families of contact lines on gear tooth surfaces. Computer Methods in Applied Mechanics and Engineering 1998, 158(1-2), 23-24. https://doi.org/10.1016/s0045-7825(97)00219-3

[8] Litvin F L, Gutman Y. Methods of Synthesis and Analysis for Hypoid Gear-Drives of "Formate" and "Helixform"-Part 1. Calculations for Machine Settings for Member Gear Manufacture of the Formate and Helixform Hypoid Gears. ASME journal of mechanical design 1981, 103(1), 89-101. https://doi.org/10.1115/1.3254890

[9] Tamotsu Koga. Method for cutting paired gears having arcuate tooth trace. American, US3915060 1975.

[10] Dai Y., Yukinori A., Desheng J. Hobbing mechanism of cylindrical gear with arcuate tooth traces and experimental investigation. China Mechanical Engineering 2006, 17(7), 706-709.

[11] Rui-Tang T., Chung-Biau T. Contact characteristics of cylindrical gears with curvilinear shaped teeth. Mechanism and Machine Theory 2004, 39 (4), 905-919. https://doi.org/10.1016/j.mechmachtheory.2004.04.006

[12] Tseng R T, Tsay C B. Mathematical model and undercutting of cylindrical gears with curvilinear shaped teeth. Mechanism and Machine Theory 2001, 36(11), 189-202. https://doi.org/10.1016/s0094-114x(01)00049-0

[13] Tseng J T, Tsay C B. Mathematical model and surface deviation of cylindrical gears with curvilinear shaped teeth cut by a hob cutter. Journal of Mechanical Design 2005, 127(5), 271-277. https://doi.org/10.1115/1.1876437

[14] Wu W., Song A. Wang Z. Stress analysis of the involute arc cylindrical gear. Chinese Journal of Mechanical Transmission 2010, (34), 38-44.

[15] Wu W., Song A. Wang Z. Research on the tooth root stress of the involute arc cylindrical dear. Chinese Journal of Machinery Design \& Manufacture 2009, (11), 227-229.

[16] Xiao Huajun, Hou Li, Dong Lu. Mathematical modeling of rotary cutter arc tooth line of cylindrical gear shaped by origin face of rotary cutter. Journal of Sichuan University 2013, 45(3), 197-199.

[17] Wang Shaojiang, Hou Li, Dong Lu. Modeling and strength analysis of cylindrical gears with curvilinear shape teeth for manufacture. Journal of Sichuan University 2012, 44 (2), 210-215.

[18] Fuentes-Aznar, A., Ruiz-Orzaez, R., Gonzalez-Perez, I. Comparison of spur, helical and curvilinear gear drives by means of stress and tooth contact analyses. Meccanica 2017, 52(7), 1721-1738. https://doi.org/10.1007/s11012-016-0515-y

[19] Zhang X, Xie Y, Tan X. Design, meshing characteristics and stress analysis of cylindrical gears with curvilinear tooth profile. Transactions of Famena 2016, 40 (1), 27-44.

[20] Chen, Y. C., C. C. Lo. Contact stress and transmission errors under load of a modified curvilinear gear set based on finite element analysis. ARCHIVE Proceedings of the Institution of Mechanical Engineers Part C Journal of Mechanical Engineering Science 2015, 203-210 (229.2),191-204. https://doi.org/10.1177/0954406214532907

[21] Fuentes, A., Ruiz-Orzaez, R., Gonzalez-Perez, I. Computerized design, simulation of meshing, and finite element analysis of two types of geometry of curvilinear cylindrical gears. Computer Methods in Applied Mechanics \& Engineering 2014, 272 (2), 321-339. https://doi.org/10.1016/j.cma.2013.12.017

[22] Zhao F., Hou L., Duan Y. Research on the forming theory analysis and digital model of circular arc gear shaped by rotary cutter. Journal of Sichuan University: Engineering Science Edition 2016, 48(6), 119 125. 
[23] Litvin F. L. Gear geometry and applied theory. PTR Prentice Hall Press 1994.

[24] Wen B. C. Mechanical design handbook. China Machine Press 2018.

[25] Han D, Jinyuan T, Wen S, et al. An innovative determination approach to tooth compliance for spiral bevel and hypoid gears by using double-curved shell model and Rayleigh-Ritz approach. Mechanism and Machine Theory, 2018, 130, 27-46. https://doi.org/10.1016/j.mechmachtheory.2018.08.009

[26] Ma, D. Q., Ye, Z. H., Yang, H. Tooth surface reconstruction and tooth profile geometric analysis of circular arc tooth trace cylindrical gears. Transactions of Famena 2019, 43(1), 29-44.

https://doi.org/10.21278/tof.43103

Submitted: $\quad 18.4 .2020$

Accepted: $\quad 03.12 .2020$
Dengqiu Ma

a. School of Mechanical and Electrical Engineering, Lanzhou University of Technology Lanzhou, China;

$b$. School of Engineering and Technology, Zunyi Normal College, Zunyi, China

Yongping Liu (corresponding author)

School of Mechanical and Electrical

Engineering, Lanzhou University of

Technology, Lanzhou, China

Zhenhuan Ye

School of Engineering and Technology, Zunyi Normal College, Zunyi, China

Yongqiao Wei

School of Mechanical and Electrical Engineering, Lanzhou University of Technology, Lanzhou, China

Jing Liu

School of Engineering and Technology, Zunyi Normal College, Zunyi, China 